\title{
Gelation versus liquid crystal phase transitions in suspensions of plate-like particles
}

\author{
By M. C. D. Mourad, J. E. G. J. Wijnhoven, D. D. van' 't Zand, \\ D. VAN DER BeEK AND H. N. W. LeKKerkerker* \\ Van 't Hoff Laboratory for Physical and Colloid Chemistry, Debye Institute, \\ Utrecht University, Padualaan 8, 3584 CH Utrecht, The Netherlands
}

Gelation is a common effect in aqueous suspensions of charged colloidal clay platelets at concentrations as low as $1 \mathrm{wt} \%$. However, in systems of charged gibbsite $\left[\mathrm{Al}(\mathrm{OH})_{3}\right]$ platelets, gelation can be delayed to concentrations as high as $50 \mathrm{wt} \%$ depending on the ionic strength. We investigated the phase behaviour of this system approaching the state of gelation in the delicate region between attractive and repulsive states that originate from competition between Coulomb repulsion and van der Waals attraction. As a function of the ionic strength, isotropic-nematic, nematic-columnar and isotropiccolumnar phase separations were observed. Moreover, compression by gravitational forces allowed us to observe phase separation that is arrested by gelation in the homogeneous suspensions.

Keywords: colloid; suspension; gelation; phase transition; nematic; columnar

\section{Introduction}

The isotropic-nematic phase transition in a system of charged colloidal platelets was reported by Irving Langmuir as early as 1938. In a remarkable paper, he reported on sols of California bentonite clay particles that after standing for several hundreds of hours, separated into two distinct phases (Langmuir 1938). The phase-separated system can be identified as an isotropic phase (I) in equilibrium with a nematic liquid crystal phase $(\mathrm{N})$. This separation was found to occur for sols containing between 2.0 and $2.2 \mathrm{wt} \%$ clay particles. Langmuir refers to his clay as 'Californian bentonite'. The name bentonite is nowadays generally used to indicate montmorillonite, a dioctahedral smectite clay. However, Langmuir did not have a montmorillonite in hand. From the adjective he uses, we know that he was using the clay from California. From the elemental analysis, provided by Langmuir, it is clear that he worked with the trioctahedral smectite clay, named hectorite in 1941 after its finding place Hector, California (Bihannic et al. 2001).

Inspired by the experiments of Langmuir (1938) on plate-like clay particles, Onsager (1949) put forward his theoretical explanation for the I-N transition in suspensions of plate-like particles. The spontaneous orientational ordering of the

* Author for correspondence (h.n.w.lekkerkerker@chem.uu.nl).

One contribution of 18 to a Discussion Meeting Issue 'New directions in liquid crystals'. 


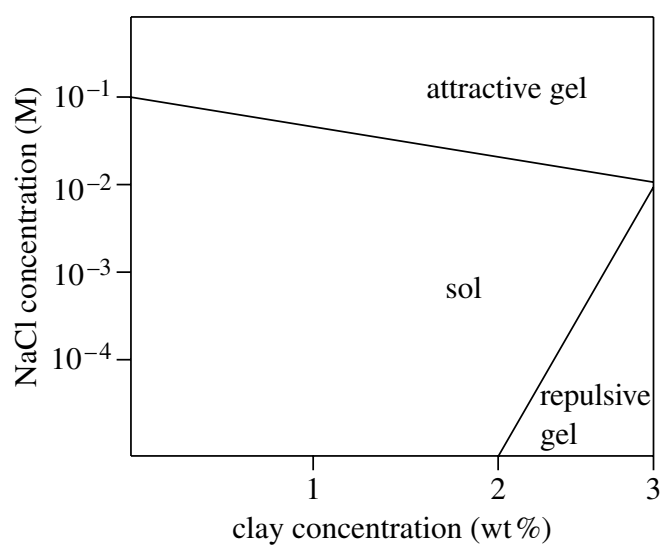

Figure 1. Schematic representation of the sol-gel diagram for clay suspensions.

particles is caused by a strongly anisotropic excluded volume interaction between them that favours the parallel alignment of the particles working against the orientational entropy, which favours the opposite, i.e. random orientations of the particles.

This seminal idea of Onsager has been confirmed by computer simulations (Frenkel \& Eppenga 1982; Veerman \& Frenkel 1992; Bates 1999; Zhang et al. 2002) albeit that, as Onsager himself already realized, his theory describes concentrated suspensions of plate-like particles qualitatively rather than quantitatively. The computer simulations (Veerman \& Frenkel 1992) moreover demonstrated that for plate-like particles of finite thickness, a hexagonal columnar phase ( $\mathrm{Col}$ ) becomes stable thermodynamically at sufficiently higher concentrations. Specifically, these simulations show that hard platelets undergo either the $\mathrm{I}-\mathrm{N}$ transition and subsequently the $\mathrm{N}-\mathrm{Col}$ transition or directly the I-Col phase transition, depending on the diameter-to-thickness ratio.

On the experimental side, quite surprisingly, the I-N phase transition as reported by Langmuir, has been observed again neither in the natural plate-like clay systems (Gabriel et al. 1996; Michot et al. 2004) nor in the synthetic hectorite and laponite (Mourchid et al. 1995, 1998; Gabriel et al. 1996); gelation is found instead. Typically at solid contents above $2-3 \%$ clay dispersions become gel-like with the appearance of a yield stress and viscoelastic properties. Rheological studies (Abend \& Lagaly 2000) have revealed that the yield stress depends strongly on the salt concentration and that it decreases to a minimum around 1-10 mM salt (depending on the kind of clay). One distinguishes a repulsive gel and an attractive gel (Abend \& Lagaly 2000; Tanaka et al. 2004) separated by a gap of sol states. This is shown schematically in figure 1.

Apparently, in clay suspensions, the liquid crystal phases are buried behind the gel lines and hence are not observed as thermodynamic equilibrium states. In recent years, nematic (Liu et al. 2003; van der Beek \& Lekkerkerker 2003, 2004; Fossum et al. 2005), columnar (Brown et al. 1998; van der Beek \& Lekkerkerker 2004) and lamellar (Wang et al. 2005) liquid crystal phases have been observed in suspensions of synthetic, charged, plate-like particles. These 
mineral liquid crystals (Gabriel \& Davidson 2000; Davidson \& Gabriel 2005) are quite promising as they have the potential of enhanced electrical, optical and magnetic properties and thermal stability compared to organic liquid crystal materials.

Here, we investigate the competition between gelation and liquid crystal phase transitions in suspensions of charged colloidal gibbsite platelets as a function of salt concentration. The suspensions of charged colloidal gibbsite platelets form nematic and columnar liquid crystal phases (van der Beek \& Lekkerkerker 2003). By variation of the ionic strengths, the effective aspect ratio (thicknessto-diameter ratio) of the platelets can be tuned, which in turn determines the liquid crystal phase behaviour. At the same time, the gel line shifts changing the observation window for liquid crystal phases.

\section{Experimental methods}

The colloidal gibbsite platelets were synthesized following a preparation method as described in van der Kooij \& Lekkerkerker (1998), Wierenga et al. (1998) and van der Beek \& Lekkerkerker (2004). To demineralized water $\left(1 \mathrm{dm}^{3}\right)$, hydrochloric acid ( $\mathrm{HCl} 0.09 \mathrm{M}, 37 \%$, Merck), aluminium sec-butoxide $(0.08 \mathrm{M}$, $95 \%$ or more, Fluka Chemika) and $0.08 \mathrm{M}$ aluminium isopropoxide (98\% or greater, Acros Organics) are added. The mixture is stirred for 10 days and subsequently heated in a polyethylene bottle in a water bath at $85^{\circ} \mathrm{C}$ for $72 \mathrm{~h}$. After cooling to room temperature, the dispersion is dialysed for one week against a continuous flow of demineralized water in regenerated cellulose tubes (Visking, MWCO 12 000-14000). In order to reduce polydispersity and concentrate the particles, dispersions are centrifuged at $1100 \mathrm{~g}$ until the supernatant is bluish (overnight, 15-20 h). As a result, hexagonal platelets of average diameter $\langle D\rangle=$ $210 \mathrm{~nm}$ (19\% polydispersity) are obtained (Wijnhoven 2005). The sediment is redispersed in water and added to a fresh aluminium alkoxide mixture with the same contents as described earlier. The heating procedure is repeated in order to grow the particles while stirring the mixture and is followed by dialysing and centrifuging as described earlier. The resulting dispersion contains particles (figure 2) of $\langle D\rangle=270 \mathrm{~nm}$ (18\%) and thickness $15 \mathrm{~nm}$ (48\%) as determined by transmission electron microscopy (120 kV Tecnai 12, FEI Company) and atomic force microscopy (Digital instruments), respectively.

The surface of the gibbsite particles is treated by hydrolysis of aluminium chlorohydrate $(\mathrm{ACH})$ to enhance the surface charge as determined by Hernandez (1998). To gibbsite $(1 \mathrm{~g})$ in suspension, ACH $(0.625 \mathrm{~g})$ is added. After 3 days, the excess ACH is removed by two sequences of sedimentation and redispersion in $\mathrm{NaCl}$ solutions in order to bring the samples to the intended ionic strength and concentration. Then, samples are transferred into flat borosilicate glass capillaries (height $\times$ width $\times$ thickness $100 \times 10 \times 1 \mathrm{~mm}^{3}$, Vitrocom) that are subsequently flame sealed. The resulting samples were made at low, intermediate and high ionic strength $\left(10^{-4}, 10^{-3}\right.$ and $10^{-1} \mathrm{M} \mathrm{NaCl}$, respectively) for concentrations of 300,400 and $500 \mathrm{~g} \mathrm{dm}^{-3}$.

The samples were stored in a thermostatted room and inspected for at least one month after preparation, both under direct illumination and between crossed polarizers, investigating the appearance and the evolution of birefringence and 


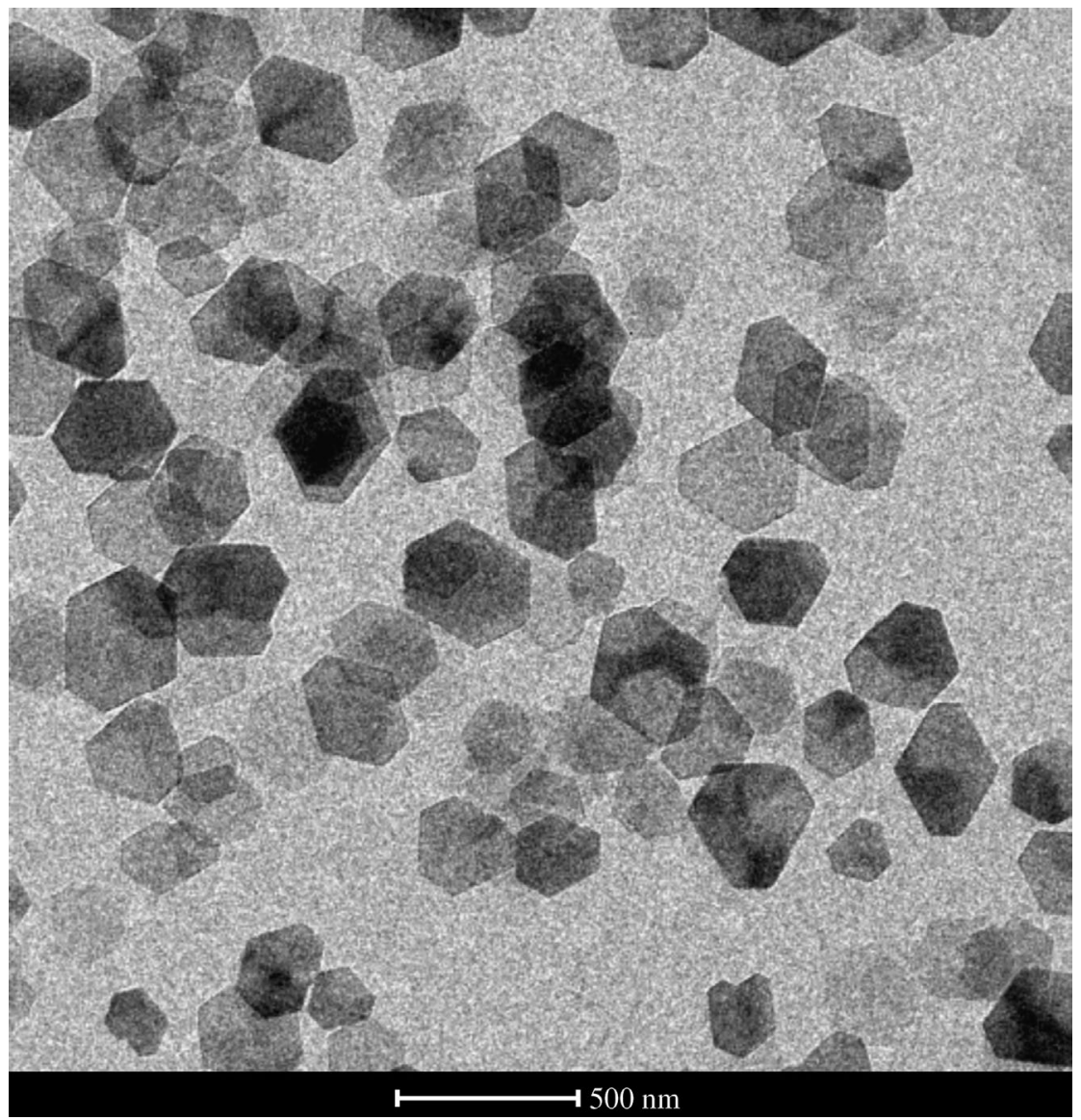

Figure 2. Transmission electron micrograph of the hexagonal gibbsite platelets used in this study.

iridescence (optical Bragg reflections). Phases that show only birefringence are recognized as nematic, whereas phases that show both birefringence and iridescence are identified as columnar.

\section{Results}

(a) Low ionic strength: $10^{-4} \mathrm{M} \mathrm{NaCl}$

At an ionic strength of $10^{-4} \mathrm{M} \mathrm{NaCl}$ and a particle concentration of $200 \mathrm{~g} \mathrm{dm}^{-3}$, gibbsite was found to form a birefringent gel (Wijnhoven et al. 2005 ) that remains unimpaired by gravity even after 2 years (figure 3 ). At a concentration of $100 \mathrm{~g} \mathrm{dm}^{-3}$, separation into an isotropic phase and a small sediment takes place within a few days. After 1 year, the sediment has grown slightly due to gravitational settling and Bragg reflecting spots have appeared, revealing the presence of positional order in the form of the columnar phase (figure 4). 


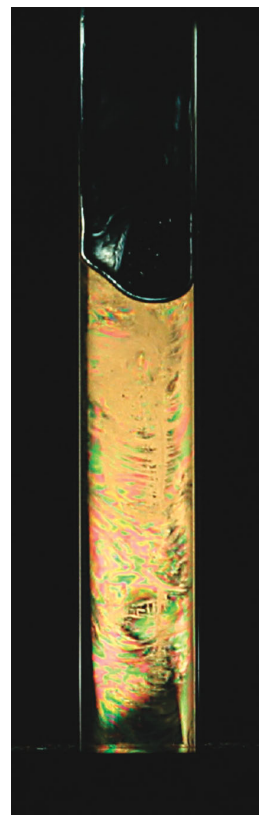

Figure 3. Photograph of $200 \mathrm{~g} \mathrm{dm}^{-3}$ gibbsite at $10^{-4} \mathrm{M}$ between crossed polarizers, 2.5 years after preparation.

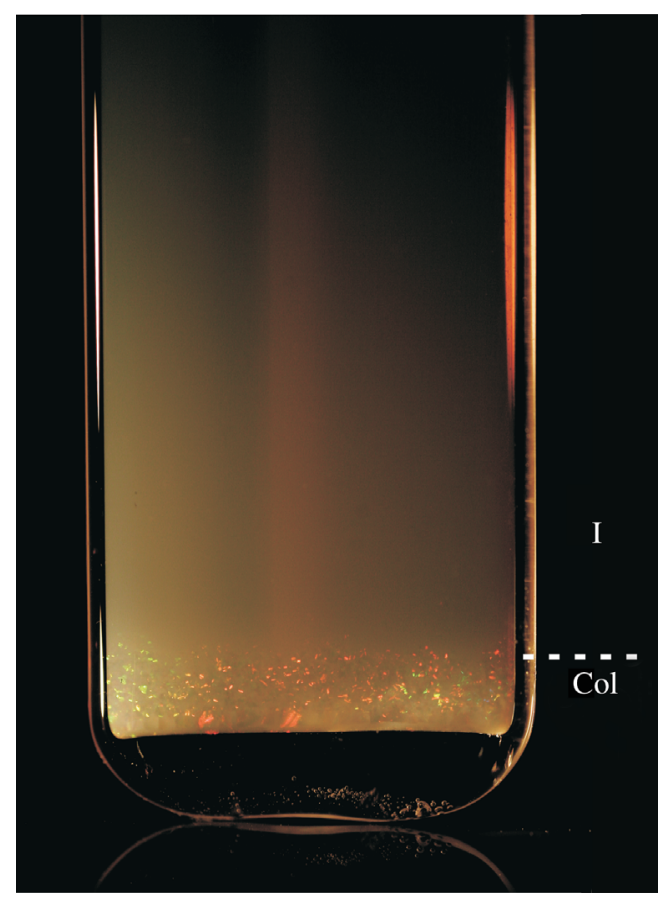

Figure 4. Photograph of a $100 \mathrm{~g} \mathrm{dm}^{-3}$ sample at $10^{-4} \mathrm{M}$, illuminated by white light. The top part is isotropic (I), while the bottom part is columnar $(\mathrm{Col})$ as is revealed by the presence of Bragg reflections. The photograph is taken 2.5 years after preparation. 


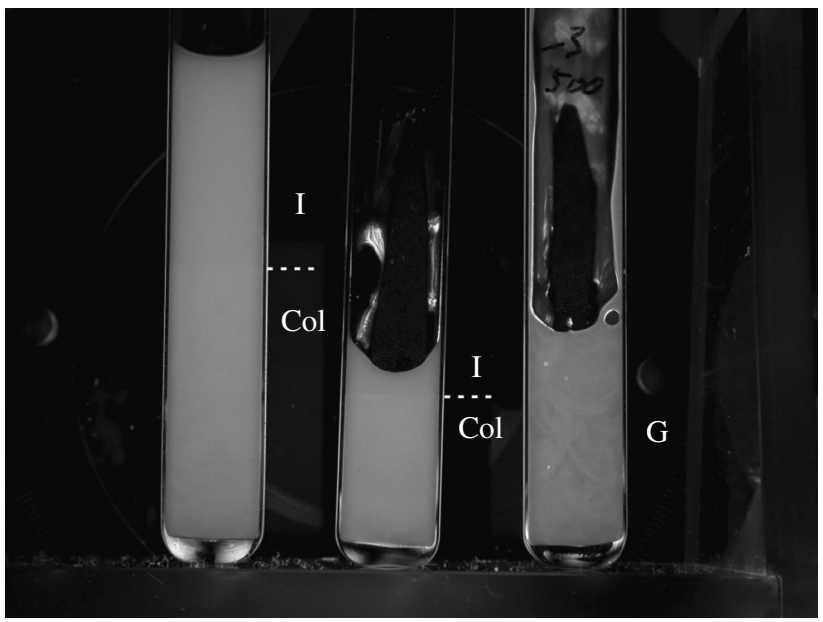

Figure 5. Photograph of $300 \mathrm{~g} \mathrm{dm}^{-3}$ (left), $400 \mathrm{~g} \mathrm{dm}^{-3}$ (middle) and $500 \mathrm{~g} \mathrm{dm}^{-3}$ (right) samples at $10^{-3} \mathrm{M}$, taken between crossed polarizers, one month after preparation. I refers to the isotropic phase, Col, the columnar phase and $\mathrm{G}$, the gel phase.

\section{(b) Intermediate ionic strength: $10^{-3} \mathrm{M} \mathrm{NaCl}$}

At $10^{-3} \mathrm{M} \mathrm{NaCl}$, Wijnhoven et al. (2005) observed that a $200 \mathrm{~g} \mathrm{dm}^{-3}$ gibbsite suspension initially forms a transient gel that phase separates within three weeks into an isotropic and columnar phase. At 300 and $400 \mathrm{~g} \mathrm{dm}^{-3}$, suspensions were found to act similarly; the initial birefringent gel-like suspension (as it is perceived from the birefringence pattern) phase separates within 10 days into an isotropic and a birefringent phase (figure 5). The lower phase shrinks further in time as an effect of gravity, as was observed earlier for $200 \mathrm{~g} \mathrm{dm}^{-3}$. The Bragg reflecting spots can be observed throughout the lower phase of 300 and $400 \mathrm{~g} \mathrm{dm}^{-3}$ (figure 6). At $500 \mathrm{~g} \mathrm{dm}^{-3}$, the suspension ends up in a birefringent gel that remains intact in time despite gravity (figure 5).

\section{(c) High ionic strength: $10^{-2} \mathrm{M} \mathrm{NaCl}$}

At $10^{-2} \mathrm{M} \mathrm{NaCl}$, a suspension of $200 \mathrm{~g} \mathrm{dm}^{-3}$ gibbsite was found to phase separate into three phases during one week: isotropic, nematic and sediment that develops Bragg reflecting spots after a few months (Wijnhoven et al. 2005).

Starting off from what seems to be a birefringent gel, suspensions of 300 and $400 \mathrm{~g} \mathrm{dm}^{-3}$ phase separate into an isotropic (upper) phase and a birefringent (lower) phase within one week (figure 7). At the same time, the first signs of sedimentation are clearly visible at the top of the isotropic phase. After one month, Bragg reflections become visible in the lower phase (figure 8). At $500 \mathrm{~g} \mathrm{dm}^{-3}$, two phases can be distinguished after a few days; the upper phase has the same threaded (nematic) texture as the lower phases at 300 and $400 \mathrm{~g} \mathrm{dm}^{-3}$, the lower phase has a homogeneous texture (figure 7). Already, after two weeks, Bragg reflections become visible in the lower phase (figure 8). 


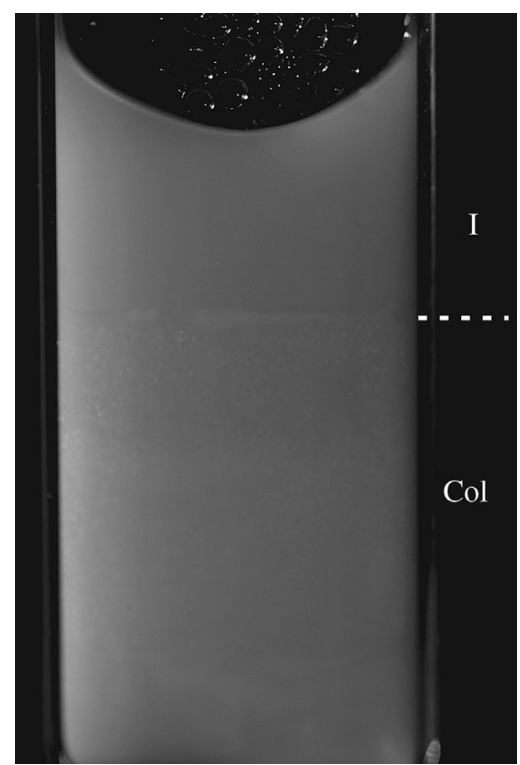

Figure 6. Photograph of $400 \mathrm{~g} \mathrm{dm}^{-3}$ sample at $10^{-3} \mathrm{M}$ illuminated by white light. Iridescence is visible in the lower phase, revealing its columnar nature.

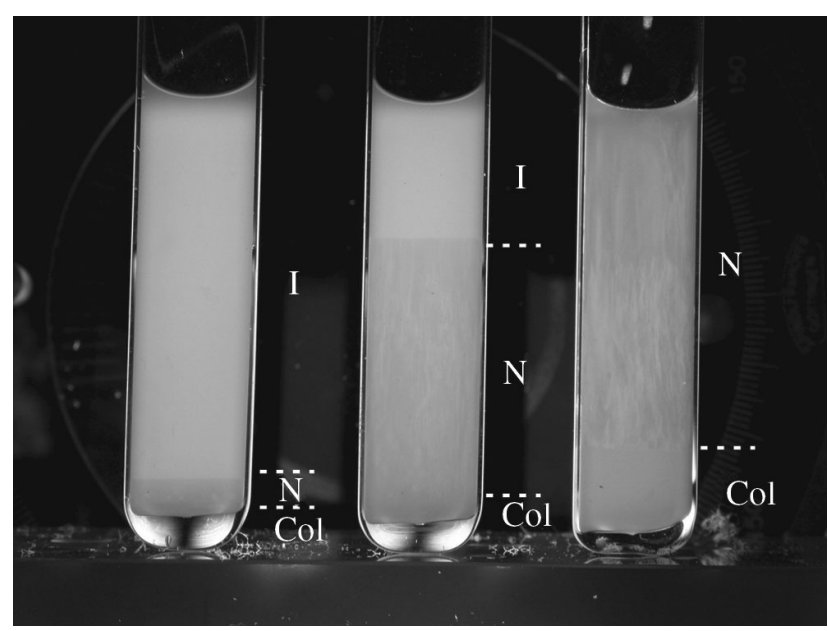

Figure 7. Photograph of $300 \mathrm{~g} \mathrm{dm}^{-3}$ (left), $400 \mathrm{~g} \mathrm{dm}^{-3}$ (middle) and $500 \mathrm{~g} \mathrm{dm}^{-3}$ (right) samples at $10^{-2} \mathrm{M}$, taken between crossed polarizers, one month after preparation. I refers to the isotropic phase, $\mathrm{N}$, the nematic phase and $\mathrm{Col}$, the columnar phase.

\section{Discussion and conclusions}

In suspensions of charged gibbsite platelets, liquid crystal phases can be observed within a few days after preparation of the samples. Both the sharp interfaces as well as the textures that are observed when the samples are placed between cross polarizers indicate phase separation. Gelation is delayed to much higher concentrations than for suspensions of charged colloidal clay 


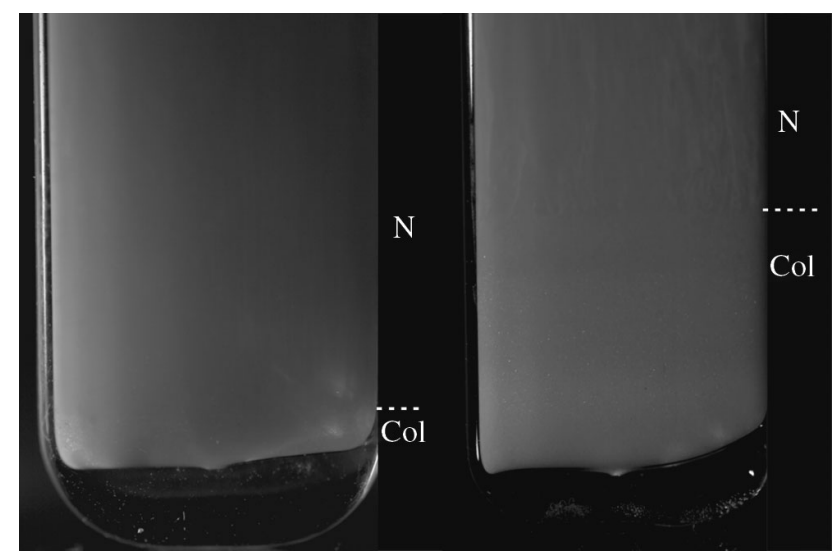

Figure 8. Photograph of $400 \mathrm{~g} \mathrm{dm}^{-3}$ (left) and $500 \mathrm{~g} \mathrm{dm}^{-3}$ (right) samples at $10^{-2} \mathrm{M}$ illuminated by white light. Iridescence can be seen in the lower phases revealing their columnar nature.

platelets (Mourchid et al. 1995; Gabriel et al. 1996; Mourchid et al. 1998; Abend \& Lagaly 2000; Michot et al. 2004). The increase in the critical gelation concentration with ionic strength confirms that we are investigating the repulsive regime in which interactions depend on the Debye screening length. At longer time-scales (weeks to months), compression by gravity leads to the modification of phase volumes and more interestingly to the appearance of new phases even where they would be unattainable due to gelation. For example, at $10 \mathrm{wt} \%$ in $10^{-4} \mathrm{M} \mathrm{NaCl}$, a columnar phase is formed through sedimentation.

While we have not provided an explanation for the delay in the critical gelation concentration in the charged gibbsite system, it has as a consequence that the theoretically predicted rich liquid crystal phase behaviour comes within experimental reach. Clearly, this opens up new avenues in liquid crystal research.

We thank Jan den Boesterd (Audiovisuele Dienst Chemie, Utrecht University) for taking some of the photographs and Hans Meeldijk (Electron Microscopy, Utrecht University). Schlumberger (Cambridge, UK) is thanked for funding the work of D.D.Z. The work of M.C.D.M., J.E.G.J.W. and D.B. was supported financially by the 'Nederlandse Organisatie voor Wetenschappelijk Onderzoek' (NWO).

\section{References}

Abend, S. \& Lagaly, G. 2000 Sol-gel transitions of sodium montmorillonite dispersions. Appl. Clay Sci. 16, 201. (doi:10.1016/S0169-1317(99)00040-X)

Bates, M. A. 1999 Influence of particle shape on the nematic-isotropic transition of colloidal platelet systems. J. Chem. Phys. 111, 1732-1736. (doi:10.1063/1.479433)

Bihannic, I., Michot, L. J., Lartriges, B. S., Vantelon, D., Labille, J., Thomas, F., Susini, J., Salomé, M. \& Fayard, B. 2001 First direct visualization of oriented mesostructures in clay gels by synchrotronbased X-ray fluorescence microscopy. Langmuir 17, 4144-4147. (doi:10.1021/la0101494)

Brown, A. B. D., Clarke, S. M. \& Rennie, A. R. 1998 Ordered phase of platelike particles in concentrated dispersions. Langmuir 14, 3129-3132. (doi:10.1021/la971294d)

Davidson, P. \& Gabriel, J.-C. P. 2005 Mineral liquid crystals. Curr. Opin. Colloid Interface Sci. 9, 377. (doi:10.1016/j.cocis.2004.12.001) 
Fossum, J. O., Gudding, E., Fonseca, D. D. M., Meheust, Y., DiMasi, E., Gog, T. \& Venkataraman, C. 2005 Observations of orientational ordering in aqueous suspensions of a nano-layered silicate. Energy 30, 873-883. (doi:10.1016/j.energy.2004.04.008)

Frenkel, D. \& Eppenga, R. 1982 Monte Carlo study of the isotropic-nematic transition in a fluid of thin hard disks. Phys. Rev. Lett. 49, 1089-1092. (doi:10.1103/PhysRevLett.49.1089)

Gabriel, J. C. P. \& Davidson, P. 2000 New trends in colloidal liquid crystals based on mineral moieties. Adv. Mater. 12, 9-20. (doi:10.1002/(SICI)1521-4095(200001)12:1<9::AID-ADMA9> 3.0. CO;2-6)

Gabriel, J. C. P., Sanchez, C. \& Davidson, P. 1996 Observation of nematic liquid-crystal textures in aqueous gels of smectite clays. J. Phys. Chem. 100, 11 139-11 143. (doi:10.1021/jp961088z)

Hernandez, J. 1998 Doctoral thesis, Université Pierre et Marie Curie.

Langmuir, I. 1938 The role of attractive and repulsive forces in the formation of tactoids, thixotropic gels, protein crystals and coacervates. J. Chem. Phys. 6, 873-896. (doi:10.1063/ $1.1750183)$

Liu, S., Zhang, J., Wang, N., Liu, W., Zhang, C. \& Sun, D. 2003 Liquid-crystalline phases of colloidal dispersions of layered double hydroxides. Chem. Mater. 15, 3240-3241. (doi:10.1021/ cm034201o)

Michot, L. J., Bihannic, I., Porsch, K., Maddi, S., Baravian, C., Mougel, J. \& Levitz, P. 2004 Phase diagrams of Wyoming Na-montmorillonite clay. Influence of particle anisotropy. Langmuir $\mathbf{2 0}$, 10 829-10 837. (doi:10.1021/la0489108)

Mourchid, A., Delville, A., Lambard, J., Lécolier, E. \& Levitz, P. 1995 Phase diagram of colloidal dispersions of anisotropic charged particles: equilibrium properties structure, and rheology of laponite suspensions. Langmuir 11, 1942-1950. (doi:10.1021/la00006a020)

Mourchid, A., Lécolier, E., Van Damme, H. \& Levitz, P. 1998 On viscoelastic, birefringent, and swelling properties of laponite clay suspensions: revisited phase diagram. Langmuir 14, 4718-4723. (doi:10.1021/la980117p)

Onsager, L. 1949 The effects of shape on the interaction of colloidal particles. Ann. NY Acad. Sci. $\mathbf{5 1}, 627-659$.

Tanaka, H., Meunier, J. \& Bonn, D. 2004 Nonergodic states of charged colloidal suspensions: repulsive and attractive glasses and gels. Phys. Rev. E 69, 031 404. (doi:10.1103/PhysRevE.69.031404)

van der Beek, D. \& Lekkerkerker, H. N. W. 2003 Nematic ordering vs. gelation in suspensions of charged platelets. Europhys. Lett. 61, 702-707. (doi:10.1209/epl/i2003-00132-1)

van der Beek, D. \& Lekkerkerker, H. N. W. 2004 Liquid crystal phases of charged colloidal platelets. Langmuir 20, 8582-8586. (doi:10.1021/la049455i)

van der Kooij, F. M. \& Lekkerkerker, H. N. W. 1998 Formation of nematic liquid crystals in suspensions of hard colloidal platelets. J. Phys. Chem. B 102, 7829-7832. (doi:10.1021/ jp981534d)

Veerman, J. A. C. \& Frenkel, D. 1992 Phase behavior of disklike hard-core mesogens. Phys. Rev. A 45, 5632-5648. (doi:10.1103/PhysRevA.45.5632)

Wang, N., Liu, S., Zhang, J., Wu, Z., Chen, J. \& Sun, D. 2005 Lamellar phase in colloidal suspensions of positively charged LDHs platelets. Soft Matter 1, 428-430. (doi:10.1039/ b512262g)

Wierenga, A. M., Lenstra, T. A. J. \& Philipse, A. P. 1998 Aqueous dispersions of colloidal gibbsite platelets: synthesis, characterisation and intrinsic viscosity measurements. Colloids Surf. A 134, 359-371. (doi:10.1016/S0927-7757(97)00224-0)

Wijnhoven, J. E. G. J. 2005 Seeded growth of monodisperse gibbsite platelets to adjustable sizes. J. Colloid Interface Sci. 292, 403. (doi:10.1016/j.jcis.2005.06.018)

Wijnhoven, J. E. G. J., van 't Zand, D. D., vanderBeek, D. \& Lekkerkerker, H. N. W. 2005 Sedimentation and phase transitions of colloidal gibbsite platelets. Langmuir 21, $10422-10427$. (doi:10.1021/la0513860)

Zhang, S.-D., Reynolds, P. A. \& van Duijneveldt, J. S. 2002 Phase behavior of mixtures of colloidal platelets and nonadsorbing polymers. J. Chem. Phys. 117, 9947-9958. (doi:10.1063/1.1518007) 


\section{Discussion}

S. T. Lagerwall (The Royal Swedish Academy of Sciences, Sweden). Ice crystals are discotic. Could it be that they form a nematic like your clay phase, but with air more or less replacing the solvent? Would it be possible that this could influence avalanche behaviour?

H. LEKKERKERKER. While ice crystals have indeed a hexagonal shape, they are of such a size that they do not undergo Brownian motion and in that sense will not display an entropically driven columnar phase. However, in the pursuit of perfect packing, it may well be that the columnar arrangement is favoured and this in turn will influence the granular flow properties. Whether this could go as far as influencing avalanche behaviour I do not speculate.

M. WiLson (Department of Chemistry, University of Durham, UK). In the phase diagram of Veerman/Frenkel there is a small area corresponding to a cubatic phase. Is this phase accessible with experiment and are there interesting interference properties expected?

H. LeKKERKERKER. The cubatic phase indeed occupies a small part in the phase diagram of Veerman and Frenkel, and therefore it is not easy to access. Moreover, one must have a probe that is sensitive to the cubatic order parameter, which is also not easy. In any case, we have not obtained evidence for it but, quite honestly, we did not hunt for it.

S. KLeIN (Hewlett Packard, Bristol, UK). Different clays have different aspect ratios. Have you ever tried to measure how flexible these clays are, for example laponite compared to bentonite?

H. LEKKERKERKER. Our gibbsite particles, having a thickness of about $10 \mathrm{~nm}$ (or more) are, as far as we observe them in electron microscopy, completely rigid. The experimental evidence for laponite as far as this issue is concerned is limited, but indicates that these small (diameter about $30 \mathrm{~nm}$ ) platelets are rigid. On the other hand, bentonite in exfoliated form is thin (thickness $1 \mathrm{~nm}$ ) and can be very large in diameter (order $1 \mu \mathrm{m}$ ) and is certainly flexible. It is tempting to assume that the non-occurrence of the isotropic-to-nematic phase transition in bentonite is related to its flexibility. However, laponite, which is rigid, is even more prone to gelation than bentonite. Nevertheless, the gibbsite example shows that particles with a thickness of the order of $10 \mathrm{~nm}$ do exhibit the isotropicto-nematic phase transition and my own feeling is that we have to look in this direction.

C. V. Kulkarni (Department of Chemistry, Imperial College London, UK). How do the synthetic analogues of natural clays compare with Al hydroxide and its prospectus?

H. LEKKERKERKER. Laponite, which is a synthetic variety of hectorite, gels very quickly and does not show any sign of the isotropic-nematic phase transition. On the other hand, Dejun Sun and co-workers (Liu et al. 2003; Wang et al. 2005) do observe nematic and lamellar phases in colloidal suspensions of synthetic layered double hydroxides. 